\title{
SIGNIFICANT DRUG INTERACTIONS AMONG INTENSIVE CARE UNIT PATIENTS
}

\author{
YULIS HAMIDY M*, DINA FAUZIA
}

Department of Pharmacology, Faculty of Medicine, University of Riau, Indonesia. Email: yulis.hamidy@gmail.com

\author{
Received: 06 November 2016, Revised and Accepted: 24 January 2017
}

\section{ABSTRACT}

Objective: Drug interaction is one factor that contributes to drug-related problems. The hospitalized patients in intensive care units (ICU) have a higher risk for developing drug interactions. The purpose of this study was to evaluate the potency of significant drug interactions in ICU patients.

Methods: Drug-drug interactions from patient's medical records from ICU of Arifin Achmad General Hospital in Pekanbaru, Province of Riau, Indonesia at period of July to December 2015 were assessed. Drug Interaction Checker (Medscape) software was used to identify potential drug interactions.

Results: This study included 28 ICU patients (mean age, 48 years) who had the potency to drug interactions based on the software. Of these, $29 \%$ were male and $71 \%$ were female patients. The number of drugs that were given to patients was 3-13 drugs (average 7 drugs per patient). There were 122 potential drug-drug interactions found in this study, including $43 \%$ potency of minor or non-significant, $52 \%$ potency of significant, $3 \%$ potency of serious, and $2 \%$ potency of contraindicated drug interactions. A total of $67 \%$ were pharmacodynamics and $33 \%$ were pharmacokinetics interactions. Dexamethasone, ketoprofen, ketorolac, furosemide, nifedipine, and enoxaparin were among drugs with the highest frequency of potential drug interactions.

Conclusion: Significant drug-drug interactions were prevalent in the ICU patients. This may be due to the complexity of the pharmacotherapies administered. The health professionals who provide care to these patients should be aware to identify and prevent possible drug events.

Keywords: Significant drug interactions, Intensive care unit, Potency, Drug-related problems.

(C) 2017 The Authors. Published by Innovare Academic Sciences Pvt Ltd. This is an open access article under the CC BY license (http://creativecommons. org/licenses/by/4. 0/) DOI: http://dx.doi.org/10.22159/ajpcr.2017.v10s2.19482

\section{INTRODUCTION}

Concurrent administration of drugs is one factor that can alter the response to drugs. The drug interactions may be beneficial or harmful, depending on various factors related to the medication, the patient or the conditions under which the medication is used [1]. Beneficial interactions can increase efficiency or allow the reduction of the dose. The harmful interactions can increase the incidence of adverse reactions and cause a reduction of the effects or results contrary to those expected [2]. Studies have shown a positive correlation between many different drugs and drug interactions. The prevalence of potential drug interactions is $3-5 \%$ in patients who receive 3-10 drugs and will increase to $20 \%$ when they are given 10-20 drugs [3].

There are several mechanisms by which drugs may interact, but most can be categorized as pharmacokinetic interactions, pharmacodynamics interactions, or combined interactions. Knowledge of the mechanism by which a given drug interaction occurs is clinically useful, since the mechanism may influence both the time course and the methods of preventing the interaction [4].

The hospitalized patients in intensive care unit (ICU) have a higher risk for developing drug interactions than patients from other care units. In addition to multiple drugs therapy, patients in the ICU presented a risk due to the severity of disease and organ failure. Drug interactions contribute to the incidence of adverse reactions in ICU and often constitute an unrecognized complication in pharmacotherapy. The prevalence of potential drug interactions in the ICU ranged from $44.3 \%$ to $86 \%[5,6]$.

The purpose of this study was to evaluate the potency of significant drug interactions in hospitalized patients at the ICU of Arifin Achmad General Hospital in Pekanbaru, Province of Riau, Indonesia. This study is useful to circumvent adverse drug reaction and to increase drug efficacy.

\section{METHODS}

This was a prospective study conducted in the ICU of Arifin Achmad General Hospital in Pekanbaru, Province of Riau, Indonesia. The medical records of patients, hospitalized in the ICU from July to December 2015, were included in the research. The characteristic information and main diagnosis were extracted from the patients' medical records. Information regarding medications administered was collected from the medical prescription documentation. Only patients aged over 18 years old were included in this study. We constructed a specific instrument to assist in data collection. Using this data collection instrument, information was collected from patients, including age, gender, primary diagnoses (according to the International Statistical Classification of Diseases and Related Health Problems [ICD 10]), and information about administered drugs.

Potential drug interactions are interactions that could theoretically occur during patient's pharmacotherapy treatment, and which may or may not be clinically manifested. In the present investigation, the terminology "drug interaction" will be used to refer to the area that includes drug-drug interaction.

For the identification of potential drug-drug interactions, Drug Interaction Checker (Medscape) software was used. This software has the adequate sensitivity to detect drug interactions in the hospital. The Drug Interaction Checker software provides information on clinical outcomes or adverse drug reactions resulting from the interaction, and characterizes the mechanism of action. It classifies the interactions in relation to severity in four categories (minor or non-significant, significant, serious, and contraindicated). The mechanisms underlying the interaction were classified into pharmacokinetic and pharmacodynamic.

The data were stored in Microsoft ${ }^{\circledR}$ Excel 2010. Descriptive analysis was performed using frequency distribution for the categorical variables. The study was approved by the Ethics Committee of Faculty of Medicine 
University of Riau (No: 62/UN.19.5.1.1.8/UEPKK/2015) and consistent with the standards of ethics in research involving humans.

\section{RESULTS}

This study included 39 medical records from patients who hospitalized in the ICU, and of these, 28 ICU's patients had the potency to drug interactions based on the software, $20(71 \%)$ of which were from female patients. The mean age was 48 years $( \pm 19.0)$, with a minimum age of 21 and a maximum of 85 years. The number of drugs that were given to patients was 3-13 drugs ( 7 drugs per patient in average). The most common drugs that were administered to the patients were antimicrobial, gastrointestinal, and analgesic, or antipyretics drugs. The most common diagnoses were obstetric and gynecologic, surgical, and neurological disorders.

The total number of potential drug interactions was 122 . The average number of drug interactions per patient was 4 , with a minimum 1 and a maximum of 18 drug interactions.

Table 1 presents the classification of potential drug interactions with respect to severity. The severe and moderate potential interactions (significant, serious, and contraindicated), together, accounted for $57 \%$ of the interactions.

There was a predominance of potential drug interactions with a pharmacodynamic mechanism of action, with a frequency of $82(67 \%)$. The potential drug interactions of pharmacokinetic mechanism totaled $40(33 \%)$. According to analyses of the case distribution of potential pharmacokinetic drug interactions, the metabolism process was responsible for most of the potential interactions. A pharmacokinetic interaction can be determined by more than one process

The most frequent significant drug interactions are listed in Table 2. Dexamethasone, ketoprofen, ketorolac, furosemide, nifedipine, and enoxaparin were identified as the drugs with the highest frequency of potential drug interactions.

\section{DISCUSSION}

This study showed that $72 \%$ of cases had the potency of drug interactions. Similarly, Reis and Cassiani and Carvalho et al. reported a $70 \%$ prevalence of potential drug interactions in the ICU patients $[7,8]$. However, a much lower frequency of drug interactions $(39.22 \%)$ was reported by Annisa and Abdullah in geriatric

Table 1: Classification of potential drug interactions identified in intensive care unit patients

\begin{tabular}{ll}
\hline Classification & n (\%) \\
\hline Minor/non-significant & $53(43)$ \\
Significant & $63(52)$ \\
Serious & $4(3)$ \\
Contra indicated & $2(2)$ \\
\hline
\end{tabular}

Table 2: The most frequent significant drug interactions in intensive care units patients

\begin{tabular}{ll}
\hline Drug & $\mathbf{n}(\%)$ \\
\hline Dexamethasone & $21(17)$ \\
Ketoprofen & $16(13)$ \\
Ketorolac & $10(8)$ \\
Furosemide & $9(7)$ \\
Nifedipine & $7(6)$ \\
Enoxaparin & $7(6)$ \\
Others (26 kind of drugs) & $52(43)$ \\
\hline
\end{tabular}

patients [9]. A literature review by Espinosa-Bosch et al. found that the prevalence of drug interactions in hospitalized patients in Spain and England was 15-45\% [10]. Differences in the level of sensitivity and specificity of the methodologies used in identifying the potential drug interactions may explain the discrepancy between these studies.

Although the identification of drug interactions using a software approach detects potential interactions, this does not mean that possible adverse events will manifest clinically in all patients with those potential drug interactions [8]. The software is an important tool to verify potential drug interactions, but it generally produces a high signal level that may indicate a higher prevalence of potential drug interactions [11].

The average age of subjects in this study was 48 years. This finding was similar to a study reported previously [8]. Age is an important factor in drug interactions as described by Becker et al. who found that the prevalence of drug interactions was increased from $10.5 \%$ to $19.2 \%$ in patients over 55 years. Patients over 55 years are susceptible for drugrelated problems [12].

Based on the common diagnoses, most of the patients suffer from obstetric and gynecological diseases. This condition corresponds to the characteristic of the subject (71\% were female). In contrast, Reis and Cassiani and Carvalho et al. and Bregnhøj et al. revealed that potential drug interactions occurred in patients with cardiovascular diseases $[7,8,13]$. Patients with the pathological condition have an increased risk for drug interactions through pharmacokinetic mechanism [2].

The number of drugs prescribed is one of the determinants of drug interactions. The average number of drugs that were given to patients in this study was 7 drugs. A similar result was found in a study by Bregnhøj et al. [13]. On the other hand, a Brazilian study found that the average number of drugs administered to patients was 12 drugs [7]. Polypharmacy treatment can increase the potency of drug interactions $[2,14]$. We found 122 potential drug interactions (the average number of drug interactions per patient was 4) in this study. The combined use of two or more drugs, each of which has toxic effects on the same organ, can greatly increase the likelihood of organ damage. For example, concurrent administration of two nephrotoxic drugs can produce kidney damage; even though, the dose of either drug alone may be insufficient to produce toxicity. Furthermore, some drugs can synergistically enhance the organ toxicity of other drugs even though the enhancing drug has no intrinsic toxic effect on that organ [4].

Most of the potential drug interactions were severe and moderate interactions (57\%). This finding was consistent with a study by Carvalho et al. [8]. The severity of drug interaction in the samples studied was better than in other national study, where the prevalence of moderate interaction was $89.05 \%$ [9]. Based on possible mechanisms, pharmacodynamic was more often than pharmacokinetic interactions as the underlying mechanism in this study. Potential pharmacodynamic interactions showed a significant prevalence in the present study. When drugs with similar pharmacologic effects are administered concurrently, an additive or synergistic response is usually occurred. These drugs may or may not act on the same receptor to produce such effects. Conversely, drugs with opposing pharmacologic effects may reduce the response to one or both drugs. Pharmacodynamic drug interactions are relatively common in clinical practice, but adverse effects can be minimized if clinicians understand the pharmacology of the drugs involved. In this way, the drug interactions can be prevented [4]. Changes in the volume of drug distribution and other pharmacokinetic factors can also pose a safety risk for of in these patients. The activity of cytochrome P450 and the effect of P-glycoprotein are important determinants of the pharmacokinetic processes in a significant number of drugs. They 
are also involved in the mechanisms of clinically important drug interactions in ICU [2].

A strategy to anticipate potential drug interactions is by planning the schedule of drug administration. This aspect is more easily handled when the drugs are administered in a single dose. Complexity arises with multiple schemes of drug administration [15]. However, planning the schedule has little implication in the prevention of potential pharmacokinetic interactions that involve either the metabolism process or pharmacodynamics. For these categories, the main preventive measures may include strategies, such as avoiding drug combination, adjusting the dose, or monitoring for early detection of adverse effects [8].

The most frequent drug involved in drug interactions in this study was dexamethasone. Dexamethasone is induced and metabolized by CYP3A4. Inhibitors of CYP3A4 increases plasma dexamethasone concentrations, whereas the inducers of CYP3A4 decreases plasma dexamethasone concentrations [16]. Ketoprofen was also another agent frequently identified in drug interactions in this study. The possibility of increased interaction is increased when ketoprofen is given concomitantly with highly bound drugs [17]. Another nonsteroidal anti-inflammation drug that we found in the present study was ketorolac. Ketorolac is highly bound to human plasma protein (mean 99.2\%). There is no evidence in animal or human studies that ketorolac tromethamine induces or inhibits hepatic enzymes capable of metabolizing itself or other drugs [18].

Furosemide, nifedipine, and enoxaparin also exhibited significant drug interactions. Furosemide may increase the ototoxic potential of aminoglycoside antibiotics, especially in the presence of impaired renal function. Furosemide should not be used concomitantly with ethacrynic acid due to the possibility of ototoxicity. Patients receiving high doses of salicylates concomitantly with furosemide may experience salicylate toxicity at lower doses due to competitive renal excretory sites. In addition, nephrotoxicity of nephrotoxic drugs, such as cisplatin, can be enhanced by furosemide. This usually occurs when furosemide is given in a high dose or in the presence of positive fluid balance as a result of forced diuresis during cisplatin treatment [19].

Furthermore, nifedipine is metabolized by CYP3A4. Coadministration of nifedipine with phenytoin, an inducer of CYP3A4, lowers the systemic exposure to nifedipine by approximately $70 \%$. CYP3A inhibitors, such as fluconazole, itraconazole, clarithromycin, erythromycin, nefazodone, fluoxetine, saquinavir, indinavir, and nelfinavir, can increase the exposure to nifedipine when coadministered [20]. Agents that can enhance the risk of hemorrhage should be discontinued before the initiation of enoxaparin. If coadministration is essential, it is recommended to perform close clinical and laboratory monitoring [21].

The number of samples that included in this study represents the number of patients hospitalized in the ICU of Arifin Achmad General Hospital for the period of study. The sample number is also associated with the inclusion criteria and the capacity of beds in ICU.

This study contributes significantly to the practice of critical care nursing by presenting the profile of drug interactions in the ICU's patients, building an important tool for planning and interventions for improving patient safety in ICU. It is essential to implement strategies that help the health-care team to identify potential interactions. These, in turn, would be useful to the prevention and monitoring of patients at risk of developing drug interactions.

\section{CONCLUSION}

This study showed that potency of significant drug interactions is prevalent in the ICU due to the complexity of the pharmacotherapies administered. The health professionals who provide care to these patients should be aware to identify and prevent possible drug events.

Health professionals' knowledge about drug interactions and their clinical significance could help to predict drug interactions and minimize the negative impacts through adequate monitoring, when the combination is unavoidable. This kind of attitude of the health-care team contributes to the optimization and safety of pharmacotherapy in critically ill patients.

\section{ACKNOWLEDGMENTS}

We would like to thank director of Arifin Achmad General Hospital for kindly facilitating this study and Arfianti for the correction of this manuscript.

\section{REFERENCES}

1. Smithburger PL, Kane-Gill SL, Seybert AL. Drug-drug interactions in the medical intensive care unit: An assessment of frequency, severity and the medications involved. Int $\mathrm{J}$ Pharm Pract 2012;20(6):402-8

2. Spriet I, Meersseman W, de Hoon J, von Winckelmann S, Wilmer A, Willems L. Mini-series: II. Clinical aspects. Clinically relevant CYP450-mediated drug interactions in the ICU. Intensive Care Med 2009;35(4):603-12.

3. Osterhoudt K, Penning TM. Drug toxicity and poisoning. In: Brunton LL, Chabner BA, Knollman BC, editors. Goodman \& Gilman's the Pharmacological Basis of Therapeutics. 12 $2^{\text {th }}$ ed. New York: The McGraw-Hill Companies; 2011. p. 73-87.

4. Palleria C, Di Paolo A, Giofrè C, Caglioti C, Leuzzi G, Siniscalchi A, et al. Pharmacokinetic drug-drug interaction and their implication in clinical management. J Res Med Sci 2013;18(7):601-10.

5. Lima RE, De Bortoli Cassiani SH. Potential drug interactions in intensive care patients at a teaching hospital. Rev Lat Am Enfermagem 2009; 17(2):222-7

6. Hammes JA, Pfuetzenreiter F, Silveira FD, Koenig A, Westphal GA. Potential drug interactions prevalence in intensive care units. Rev Bras Ter Intensiva 2008;20(4):349-54.

7. Reis AM, Cassiani SH. Prevalence of potential drug interactions in patients in an intensive care unit of a university hospital in Brazil. Clinics (Sao Paulo) 2011;66(1):9-15.

8. Carvalho RE, Reis AM, de Faria LM, Zago KS, Cassiani SH. Prevalence of drug interactions in intensive care units in Brazil. Acta Paul Enferm 2013;26(2):150-7.

9. Annisa N, Abdullah R. Potency of drugs interaction among geriatric patients prescribing: Retrospective study in pharmacies in Bandung. Indones J Clin Pharm 2012;1(3):96-101.

10. Espinosa-Bosch M, Santos-Ramos B, Gil-Navarro MV, Santos-Rubio MD, Marín-Gil R, Villacorta-Linaza P. Prevalence of drug interactions in hospital healthcare. Int J Clin Pharm 2012;34(6):807-17.

11. Egger SS, Drewe J, Schlienger RG. Potential drug-drug interactions in the medication of medical patients at hospital discharge. Eur J Clin Pharmacol 2003;58(11):773-8.

12. Becker ML, Visser LE, van Gelder T, Hofman A, Stricker BH. Increasing exposure to drug-drug interactions between 1992 and 2005 in people aged $>$ or $=55$ years. Drugs Aging 2008;25(2):145-52.

13. Bregnhøj L, Thirstrup S, Kristensen MB, Bjerrum L, Sonne J. Prevalence of inappropriate prescribing in primary care. Pharm World Sci 2007;29(3):109-15

14. Mouly S, Meune C, Bergmann JF. Mini-series: I. Basic science. Uncertainty and inaccuracy of predicting CYP-mediated in vivo drug interactions in the ICU from in vitro models: Focus on CYP3A4. Intensive Care Med 2009;35(3):417-29.

15. Williams NT. Medication administration through enteral feeding tubes. Am J Health Syst Pharm 2008;65(24):2347-57.

16. Horn JR, Hansten PD. Prediction of Potential Drug-Drug Interactions. Available from: http://www.pharmacytimes.com/publication/issue. [Last accessed on 2016 Oct 26].

17. Batista de Carvalho LA, Marques MP, Tomkinson J. Drug-excipient interactions in ketoprofen: A vibrational spectroscopy study. Biopolymers 2006;82(4):420-4.

18. Ulugöl A, Ozyigit F, Yesilyurt O, Dogrul A. The additive antinociceptive interaction between WIN 55,212-2, a cannabinoid agonist, and ketorolac. Anesth Analg 2006;102(2):443-7. 
19. Bates DE, Beaumont SJ, Baylis BW. Ototoxicity induced by gentamicin and furosemide. Ann Pharmacother 2002;36(3):446-51.

20. Opie LH. Drug interactions of antihypertensive agents. S Afr Fam Pract 2012;54(2):523-5.
21. Kubitza D, Becka M, Schwers S, Voith B. Investigation of pharmacodynamic and pharmacokinetic interactions between rivaroxaban and enoxaparin in healthy male subjects. Clin Pharmacol Drug Dev 2013;2(3):270-7. 